\title{
Nachwirkungseffekte an UV-empfindlichen Zählrohren
}

\author{
Von Hugo Neuert * \\ (Z. Naturforschg. 3 a, 221-225 [1948]; eingegangen am 3. März 1948)
}

\begin{abstract}
In UV-empfindlichen Zählrohren mit schwach oxydierten Kathodenoberflächen (z. B. MgO) und Alkohol- bzw. Methylalfüllung tritt nach Beanspruchung des Zählers von mehreren hundert Stößen/sec außer einer Erhöhung des Nulleffekts auch eine Erhöhung der photoelektrischen Empfindlichkeit auf. Beide nehmen in ähnlicher Weise nach der starken Beanspruchung des Zählers wieder ab. Der Effekt tritt um so stärker auf, je empfindlicher der UV-Zähler ist. Die Beobachtungen weisen darauf hin, daß die Kathode unter dem Einfluß der Entladungen im Innern des Zählers zeitweise aktiviert wird. Es wurde nachgewiesen, daß es sich dabei um eine wirkliche Empfindlichkeitssteigerung handelt, die sich auf die gesamte Kathodenoberfläche erstreckt.
\end{abstract}

Z u genauen Messungen mit Zählrohren ist die Konstanz des Nulleffektes oder zumindest die genaue Kenntnis seiner zeitlichen Änderung von großer Bedeutung. Bei Versuchen mit UV-empfindlichen Zählrohren war gefunden worden, daß der Nulleffekt keineswegs immer konstant, sondern nach dem Auftreten einer großen Zahl von Entladungen im Zählrohr auf ein Vielfaches seines normalen Wertes angestiegen war und erst allmählich wieder auf seinen alten Wert zurückging ${ }^{1}$. Gleichzeitig schien auch die photoelektrische Empfindlichkeit angestiegen zu sein. Diese Erscheinung ist hier näher untersucht worden. Die Versuche weisen darauf hin, daß die Effekte auf eine kurzzeitige Aktivierung der Zählrohrkathode als Folge der Zählrohrentladungen zurückzuführen sind.

\section{Apparatur}

UV-empfindliche Zählrohre erhält man dadurch, daß man geeignete Substanzen im Hochvakuum auf das Innere des Zählrohrmantels aufdampft ${ }^{2}$. Die physikalischen Vorgänge unterscheiden sich aber im Vergleich zu denjenigen in einer Photozelle doch erheblich. Bei den Zählrohren ist es nicht möglich, eine absolut reine Oberfläche zu bewahren (z. B. eine reine Metalloberfläche), da das Füllgas im Zählrohr immer die Kathodenschicht beeinflussen wird. Bei den hiesigen Versuchen waren die physikalischen Bedingungen nun in der folgenden Weise festgelegt:

1. Die photoempfindliche Schicht wurde wie gewöhnlich im Hochvakuum bei etwa 10-4 Torr auf das Innere von Messingzylindern von $10 \mathrm{~mm} \varnothing$ und $30-40 \mathrm{~mm}$ Länge aufgedampft. Dann wurden die

* Weil a. Rh., Marktplatz 5.

1 K. H. Lauterjung u. H. Neuert, Z. Physik 122, 266 [1944].

2 H. Kreuchen, Z. Physik 94, 549 [1935] u. 97, $625[1935]$.
Messingzylinder bei Luftzutritt in Glasrohre von $40-50 \mathrm{~mm} \varnothing$ und etwa $20 \mathrm{~cm}$ Länge eingesetzt, wobei sich auf der Oberfläche eine mehr oder weniger dünne Oxydschicht bildete.

2. Es wurden nur Füllgase mit Dampfzusatz verwendet. Außer der Mischung von $90 \mathrm{~mm}$ Ar $+10 \mathrm{~mm}$ $\mathrm{C}_{2} \mathrm{H}_{5} \mathrm{OH}$ wurde vielfach Methylal mit Argon oder Wasserstoff verwendet. Auch reines Methylal konnte verwendet werden.

3. An dem Glasrohr war gegenüber einem Längsschlitz im Zählrohr ein Quarzfenster von $2 \mathrm{~mm}$ Dicke angebracht zum Durchlassen der UV-Strahlung. Diese entstammte einer $\mathrm{Hg}$-Normallampe, deren spektrale Intensitätsverteilung bekannt war. Die spektrale Empfindlichkeit wurde mit Hilfe von Schott-UV-Glasfiltern bestimmt. Mit deren Hilfe konnten die verwendeten Zähler für $\lambda>254 \mathrm{~m} \mu$ mit einer Genauigkeit von unter $1 \%$ ohne Verwendung eines Monochromators ausgemessen werden.

4. Alle Zähler hatten Zählbereiche von mindestens 100 Volt.

5. Quantitative Untersuchungen wurden durchgeführt an Kathodenschichten von $\mathrm{Ag}, \mathrm{Cd}, \mathrm{Be}, \mathrm{Cu}, \mathrm{Mg}$, Zn. (Obwohl die Schichten alle oberflächlich oxydiert waren, sollen die Zähler doch der Kürze halber als Ag-Zähler usw. bezeichnet werden.)

6. Die Durchführung der Messungen wurde durch die Verwendung einer Direktstromanzeige-Schaltung

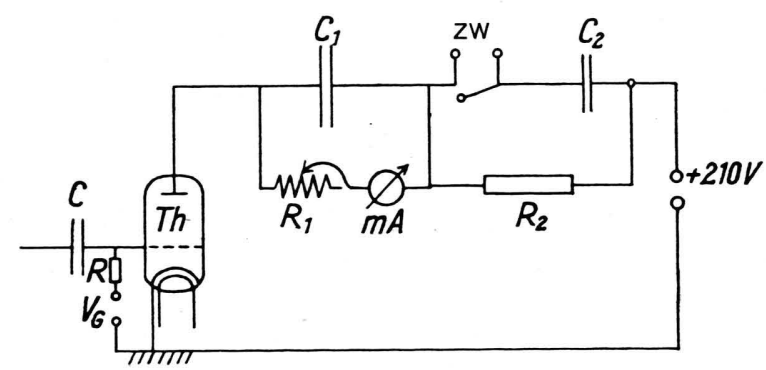

Abb. 1. Schaltbild des Strom-Meßgerätes.

$C=100 \mathrm{~cm} ; \quad C_{1}=3000 \mu \mathrm{F} ; \quad C_{2}=0,5 ; 0,1 ; 0,02 \mu \mathrm{F} ;$ $R=40 \mathrm{k} \Omega ; R_{1}=5 \mathrm{k} \Omega ; R_{2}=15 \mathrm{k} \Omega$. 
wesentlich erleichtert ${ }^{3}$ (Abb.1). Statt der Auszählung der Stoßzahlen mit einem Zählwerk konnte die mittlere Stoßzahl direkt an einem Milliampèremeter abgelesen werden. Für nicht zu große Stoßzahlen $n$ ist der Strom $i$ für $C_{1} \gg C_{2}$

$$
\begin{gathered}
i=\frac{n\left(V_{\mathrm{A}}-V_{\mathrm{B}}\right)\left(C_{2}+\tau / R_{2}\right)}{1+\frac{C_{2}+\tau / R_{2}}{C_{1}}+n R_{1}\left(C_{2}+\tau / R_{2}\right)} \\
\cdot \frac{e^{-n R_{2}\left(C_{2}+\frac{\tau-t_{0}}{R_{2}}\right)}}{1+n t_{0}}
\end{gathered}
$$

$V_{\mathrm{A}}=$ Anodenspannung,$\quad r_{\mathrm{B}}=$ Brennspannung des Thyratrons, $\tau=$ mittlere Brenndauer des Thyratrons, $t_{0}=$ mittlere Glimmzeit des Zählrohrs.

Für sehr geringe Stoßzahlen ist

$$
i=n\left(V_{\mathrm{A}}-V_{\mathrm{B}}\right) C_{2} \text {. }
$$

Erhöhung der Dunkelstoßzahl

Wird der Nulleffekt eines Zählrohrs gemessen, unmittelbar nachdem eine große Zahl von Entladungen im Zählrohr aufgetreten war, so beobachtet man häufig eine sehr erhebliche Erhöhung des Nulleffekts, der im Verlauf von Minuten wieder auf seinen Ausgangswert zurückgeht. Diese Erscheinung ist in charakteristischer Weise bei den von uns verwendeten UV-empfindlichen Zählern aufgetreten. Sie ist bereits in einer früheren Mitteilung beschrieben worden ${ }^{\mathbf{1}}$. Etwa gleichzeitig mit uns hat $\mathrm{Spat} \mathrm{z}^{4}$ dieselbe Erscheinung bei Ag-Zählern studiert. Bei seinen Zählern wurde der Normalwert erst nach Tagen wieder erreicht. Der Effekt schien nicht wesentlich von der Gasfüllung abzuhängen.

\section{Erhöhung derphotoelektrischen A usbeute}

Bei den zunächst gemeinsam mit L a u terjung durchgeführten Messungen der photoelektrischen Ausbeute ${ }^{5}$ hat dieser zuerst bemerkt, da \& auch die photoelektrische Ausbeute nach einer hohen Stoßzahl im Zähler erhöht war. Nach einigen Minuten oder Stunden hatte der Zähler seine Normalempfindlichkeit wieder erreicht. Es war naheliegend, einen Zusammenhang zwischen dieser Beobachtung und dem schon beschriebenen Effekt der Erhöhung der Dunkelstoßzahl zu ver-

3 H. Neuert, Reichsber. Physik, Heft 5 [1944].

4 W. D. B. S p a tz, Physic. Rev. 64, 236 [1943].

${ }^{5}$ H. Neuert u. K. H. L a u terjung, Reichsber. Physik, Heft 2 [1944]. muten. Aus diesem Grunde wurde die Erscheinung hier systematisch untersucht und folgende Gesetzmäßigkeiten gefunden ${ }^{6}$ :

1. Der Effekt war in starkem Maße vom Kathodenmaterial abhängig. Er trat um so deutlicher auf, je größer die UV-Empfindlichkeit der Zähler war. Die empfindlichsten Zähler waren die Mg-Zähler (etwa $1 \cdot 10^{-4} \varepsilon / h \nu$ bei $265 \mathrm{~m} \mu$ ) ; etwas weniger empfindlich waren $\mathrm{Cd}$ - und Zn-Zähler.

2. Es war kein Unterschied festzustellen, ob die hohe Stoßzahl im Zähler durch $\boldsymbol{\gamma}$-Strahlung oder durch eine UV-Bestrahlung hervorgerufen wurde. So konnte man bereits vermuten, daß der Effekt

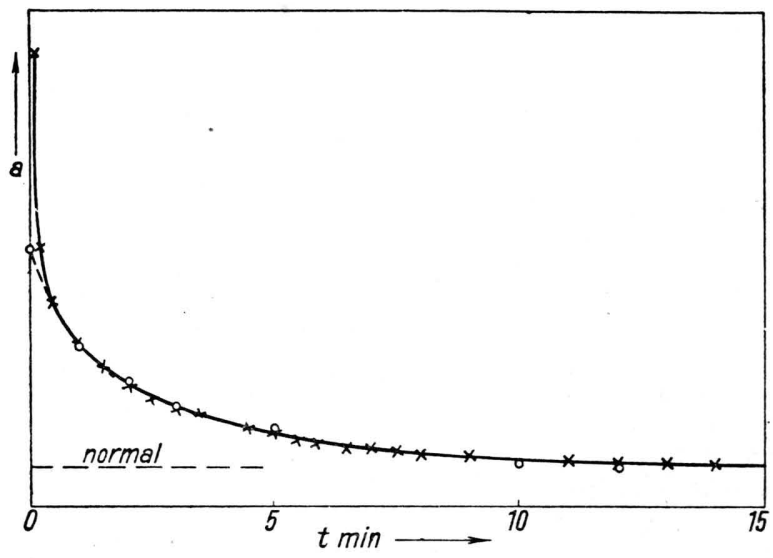

Abb. 2. Abklingen der Erhöhung der Empfindlichkeit an einem Mg-Zähler (Ar + Methylal). Ordinate: relative photoelektrische Ausbeute $a . \times \times \times$ Ausbeute für

$313 \mathrm{~m} \mu$ (1 Min. bestrahlt). o o o Tanaka-Formel.

durch Vorgänge während der Entladung innerhalb des Zählers selbst hervorgerufen wurde.

3. Abb. 2 zeigt die gemessene Erhöhung der relativen photoelektrischen Ausbeute $a$ eines MgZählers (mit Methyl + Ar) für die Linie 313 m $\mu$. Der Zähler war zuvor während einer Minute mit einigen 1000 Entladungen/sec betrieben worden. Man sieht, daß die Ausbeute unmittelbar nach der Bestrahlung auf mehr als das 10-fache des normalen Wertes angestiegen war, dann zunächst rasch, später langsam zurückging. Nach 15 Min. war der Ausgangswert annähernd wieder erreicht. Die Intensität der zur Messung verwendeten UVStrahlung war so gering, daß sie selbst keinen merklichen Effekt hervorrufen konnte.

${ }^{6}$ Die folgenden Untersuchungen konnten aus kriegsbedingten Gründen nicht mehr gemeinsam fortgesetzt werden. Die hier beschriebenen Versuche wurden in den Sommermonaten 1943 durchgeführt. Hr. L a u terjung hat später eigene Versuche ähnlicher Art unternommen (K. L a u ter jung, Naturwiss. 34, 119 [1947]. 
4. Neben der Erhöhung der photoelektrischen Ausbeute trat auch immer eine Erhöhung des Nulleffekts auf. Der Abfall der Kurven für Null-

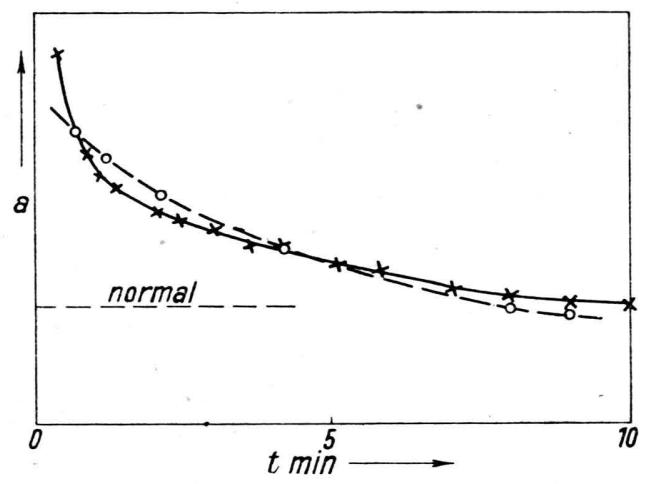

Abb. 3. Abklingen der Erhöhung der.Empfindlichkeit an einem Ag-Zähler, gemessen an 266 mu. Ordinate: relative photoelektrische Ausbeute $a$. -০-০-০- Tanaka-Formel.

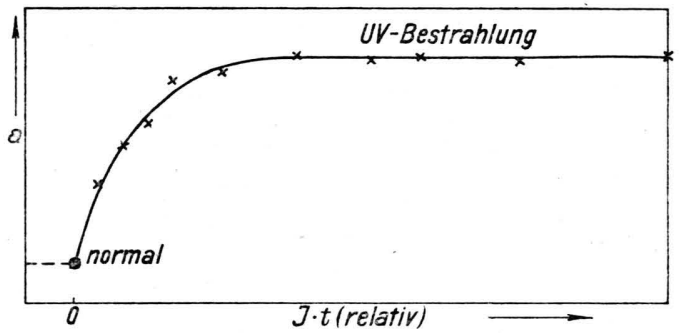

Abb. 4. Erhöhung der Empfindlichkeit bei einem MgZähler, jeweils $20 \mathrm{sec}$ nach UV-Bestrahlung. Ordinate: relative photoelektrische Ausbeute $a$.

effekt und Empfindlichkeit schienen vom gleichen Typus zu sein.

5. Der Effekt war bei allen UV-empfindlichen Zählern von derselben Art. Abb. 3 zeigt eine der Abb. 2 ähnliche Kurve für einen Ag-Zähler (gemessen an der Linie $266 \mathrm{~m} \mu$ ).

6. Es wurde qualitativ untersucht, ob der Effekt selektiv war und nur im Bereich spezieller Wellenlängen auftrat, wie das häufig bei der Aktivierung von Hochvakuumphotozellen durch Gasentladungen der Fall ist. $\mathrm{Zu}$ diesem $\mathrm{Z}$ weck wurde zunächst die Empfindlichkeit eines Mg-Zählers für mehrere Wellenlängen ausgemessen. Dann wurde der Zähler eine Minute lang mit 1000 bis 2000 Stößen/sec betrieben und die Empfindlichkeit für dieselben Wellenlängen wieder gemessen. Der Effekt schien bei allen Wellenlängen vorhanden zu sein.

7. Aber es konnte nicht einwandfrei festgestellt werden, daß die Erhöhung für alle Wellenlängen dieselbe war. Vor allem bei den Messungen im Bereich der größten Wellenlängen störte, ebenso wie bei den Bestimmungen der Grenzwellenlängen, die Erhöhung des Nulleffekts beträchtlich. Es konnte deshalb zunächst noch nicht mit Sicherheit festgestellt werden, ob mit der Erhöhung der Empfindlichkeit auch eine Verschiebung der Grenzwellenlänge nach größeren $\lambda$ hin auftrat, wie dies zu vermuten war.

8. Der Effekt stieg im Bereich mäßiger Stoßzahlen mit der Zahl der Stöße/sec und mit der Dauer der Beanspruchung des Zählers añ. Zum Studium dieses Verhaltens wurde derselbe Zähler

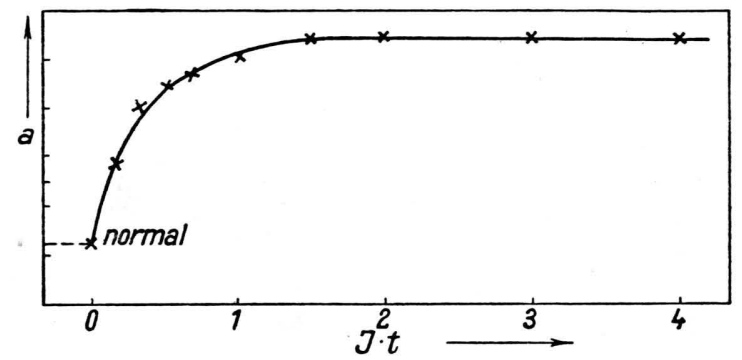

Abb. 5. Erhöhung der Empfindlichkeit bei einem AgZähler, jeweils $30 \mathrm{sec}$ nach UV-Bestrahlung. Ordinate: relative photoelektrische Ausbeute $a$

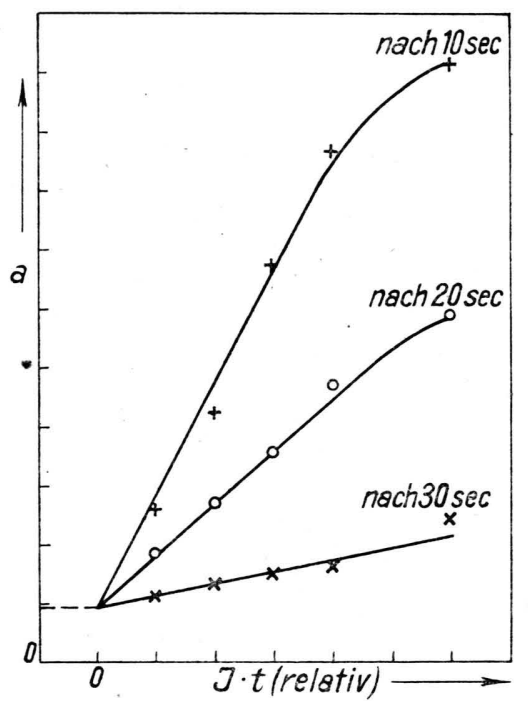

Abb. 6. Erhöhung der Empfindlichkeit bei einem $\mathrm{Mg}$ Zähler. Ordinate: relative photoelektrische Ausbeute $a$.

bei immer derselben Wellenlänge zu jeweils der gleichen Zeit (20 sec) nach der Beanspruchung ausgemessen, wobei die Intensität oder die Dauer der Belastung verändert wurde. Die Abb. 4 und 5 zeigen die Erhöhungen der Empfindlichkeiten als 
Funktion von $I \cdot t$. Für schwache bis mäßige Stoßzahlen steigt die Erhöhung der Empfindlichkeit zunächst proportional zur Beanspruchung an; nach einer gewissen maximalen, nicht zu hohen Beanspruchung tritt eine Sättigung auf. Abb. 6 zeigt den ansteigenden Teil dieser Kurven für verschiedene Zeiten nach dem Ende der starken Beanspruchung (10 sec, $20 \mathrm{sec}, 30 \mathrm{sec}$ ). Zu starke Beanspruchungen des Zählers führten leicht zu einer Dauerentladung im Zählrohr.

9. Die Intensität und die Dauer der Beanspruchung schienen auch die Art des Abklingens zu bestimmen. Stärkere Beanspruchungen hatten meist einen langsameren Abfall zur Folge.

10. $\mathrm{M} \mathrm{e} \mathrm{di} \mathrm{cu} \mathrm{s}{ }^{7}$ hat gezeigt, daß unter gewissen Bedingungen, die noch recht ungeklärt sind, ein Zählrohrstoß mit einer gewissen Wahrscheinlichkeit eine weitere spontane Entladung verursachen kann. Die Halbwertszeit eines solchen Zustandes kann bis zu mehreren Sekunden betragen. Sie hängt $a b$ von den metastabilen Zuständen des Füllgases. Es mußte nun überprüft werden, ob die zusätzlichen Stöße nach einer hohen Stoßzahl im Zähler, die als Erhöhung der Empfindlichkeit gedeutet wurden, wirkliche Stöße oder Entladungen vom Medicusschen Typus sind. Hierzu wurde folgender Versuch gemacht: Zunächst wurde der Nulleffekt eines normalen Mg-Zählers ausgemessen zu 35 Stößen/min. Nun wurde in dem Zähler durch die $\gamma$-Strahlung eines schwachen Ra-Präparats, das sich in einem wohlbekannten Abstand vom Zähler befand, eine Stoßzahl von 500/min hervorgerufen. Dann wurde das Ra-Präparat wieder entfernt und die photoelektrische Ausbeute des Zählers für die Linie $\mathrm{Hg} 313 \mathrm{~m} \mu$ mit einer schwachen UV-Intensität bestimmt, die selbst keinen Effekt hervorrufen konnte. Daraufhin wurde mit einem starken Ra-Präparat im Zähler während einer Minute eine Stoßzahl von 500 Stößen/sec erzeugt und das Präparat dann wieder entfernt. Unmittelbar nach der starken Belastung wurde kurz der etwas erhöhte Nulleffekt bestimmt und das schwache Ra-Präparat in die vorher innegehabte Stellung gebracht. Wenn man den etwas erhöhten Nulleffekt abzog, erhielt man für das schwache Präparat wieder eine Stoßzahl von $500 \mathrm{Stößen/min.} \mathrm{Schließlich} \mathrm{wurde} \mathrm{noch} \mathrm{mit}$ einer schwachen UV-Intensität die erwartete Erhöhung der photoelektrischen Ausbeute festgestellt. Daß das schwache Ra-Präparat vor und

$$
7 \text { G. Medicus, Z. Physik 103, } 76 \text { [1936]. }
$$

nach der starken Beanspruchung des Zählers die gleiche Stoßzahl ergab, ist wohl ein eindeutiger Beweis dafür, daß es sich bei dem hier untersuchten Effekt um eine wirkliche Erhöhung der Ausbeute und nicht um Medicussche Nachentladungen handelte.

11. Die Empfindlichkeitserhöhung ist gleichmäßig über die ganze Zählrohrkathode verbreitet. Dies wurde durch folgenden Versuch gezeigt: Eine Blende mit 2 Löchern von je $1 \mathrm{~mm} \varnothing$ wurde vor das Quarzfenster des Zählers gebracht. Der Abstand der beiden Löcher betrug $10 \mathrm{~mm}$. Zuerst wurde die Empfindlichkeit des Zählers für die gleiche Linie durch die beiden Löcher, d.h. an verschiedenen Stellen des Zählers, gemessen. Beide Werte waren praktisch gleich. Nun wurde Loch 1 abgedeckt und im Zähler durch Einstrahlung von UV-Licht durch Loch 2 kurze Zeit eine hohe Stoßzahl hervorgerufen. Danach wurde mit der gleichen Linie die Empfindlichkeitssteigerung durch Loch 1 und 2 gemessen, wobei die gleichen Werte gefunden wurden.

12. Zahlreiche Versuche wurden unternommen mit verschiedenen Zählern und Kathoden, um zu untersuchen, ob die Effekte auch auftreten bei gewöhnlicher Bestrahlung der Kathode, d.h. wenn das elektrische Feld des Zählers abgeschaltet ist. Es wurde bei intensiver Bestrahlung mit UVLicht durch das Quarzfenster (2 mm) kein Effekt beobachtet. Der Effekt konnte also bisher nur durch Vorgänge hervorgerufen worden sein, die in der Entladung im Zählrohr selbst ihren Ursprung hatten.

\section{Hinweis auf ähnliche Beobachtungen}

Erscheinungen ähnlicher Art sind mehrfach beobachtet worden:

a. Bei einem Zählrohr mit Kalium-Kathode und Wasserstoff als Füllgas beobachtete Christoph ${ }^{8}$ nach einer Glimmentladung eine starke Erhöhung des Nulleffekts. Dieser sank während einiger Minuten wieder auf den Ausgangswert zurück.

b. The in ${ }^{9}$ hat Versuche beschrieben, die unter ähnlichen Bedingungen, wie sie bei Zählrohren vorliegen, durchgeführt worden sind. Durch Aktivierung einer vorher nicht entgasten Al-Oberfläche mit einer $\mathrm{H}_{2}$-Glimmentladung wurde eine Steigerung der photoelektrischen Empfindlichkeit erreicht (bis zur Sättigung), die nach Ende der Entladung in wenigen Minuten wieder abgeklungen war. Dabei war sofort nach

8 O. Christoph, Ann. Physik 23, 747 [1935].

${ }^{2}$ L. P. Th e i n, Physic. Rev. 53, 287 [1938]. 
der Entladung das Füllgas weggepumpt worden; der Abfall erfolgte also im Hochvakuum.

c. Roggen und Scherre $\mathrm{r}^{\mathbf{1 0}}$ bestrahlten die Teile von Röntgenzählern mit Röntgenstrahlen, während sie auseinandergenommen waren. Danach sofort zusammengebaut, zeigten diese bei mehreren Metallen eine zunächst starke, dann allmählich abklingende Erhöhung des Nulleffekts.

d. Bei Versuchen über den statistischen Zündverzug in Gasentladungen hat $\mathrm{Pa}$ et $0 \mathrm{w}^{\mathbf{1 1}}$ gefunden, daß die Elektroden durch die Gasentladung aktiviert werden. Sie emittieren nach der kurzen Entladung einen schwachen Elektronenstrom, der ähnlich wie hier rasch abklingt. Der Effekt trat ganz besonders deutlich an den Metalloxyden $\left(\mathrm{Al}_{2} \mathrm{O}_{3}\right.$ und $\left.\mathrm{MgO}\right)$ auf. Paetow konnte zeigen, daß die Aktivierung verursacht wird durch die bei der Townsend-oder Glimmentladung entstehende kurzwellige UV-Strahlung. Ferner wurde nachgewiesen, daß die Elektronen aus dem Elektrodenmaterial und nicht aus dem Gas stammen.

e. Gü n the rschulze ${ }^{\mathbf{1 2}}$ hat ebenfalls gefunden, daß oxydierte Kathoden nach Glimmentladungen spontan Elektronen emittieren. Nach seinen Erklärungen schien die Anlagerung positiver Ionen die wirksame Ursache gewesen zu sein.

f. Die Beschießung von $\mathrm{Al}_{2} \mathrm{O}_{3}$ mit Elektronen führt nach $\mathrm{M}$ a lter ${ }^{\mathbf{1 3}} \mathrm{zu}$ einer starken Aktivierung, die dann allmählich wieder abklingt.

g. Schließlich soll noch auf Versuche von Notting $\mathrm{ham}{ }^{14}$ und von $\mathrm{Copel}$ and ${ }^{15}$ hingewiesen werden, die in Elektronenröhren in gewissen Fällen eine Änderung der Kontakt-Potentialdifferenzen zwischen Anode und Kathode fanden, nachdem die Röhre kurze Zeit stark belastet worden war (Anodeneffekt). Im Verlauf von wenigen Minuten ging die KontaktPotentialdifferenz wieder auf den alten Wert zurück.

\section{Schlußbemerkungen}

Die obigen Versuche sollten zunächst nur die Phänomenologie des Effektes aufzeigen. Über die physikalischen Ursachen des Effektes können daraus nur wenige Schlüsse gezogen werden. Man muß wohl annehmen, daß der Effekt durch das Auftreten von Ionen auf der Kathodenoberfläche hervorgerufen wird. Damit erhebt sich die Frage nach der Entstehung derselben.

10 Roggen u. Scherrer, Helv. physica Acta 15, 497 [1942].

11 H. P a e tow, Z. Physik 111, 770 [1939].

12 A. Gïntherschulze, Z. Physik 86, 778 [1933].

${ }_{13}$ L. M a l te r, Physic. Rev. 39, 183 [1932] u. 49, $78[1936]$.
Es ist zunächst naheliegend, zu vermuten, daß sich Ionen direkt aus der Entladung an den isolierenden Metalloxydteilchen der Kathodenoberfläche anlagern und dadurch örtlich hohe Feldstärken hervorrufen, die ihrerseits Anlaß zu spontaner Elektronenemission geben (Elektronenemission durch hohe Feldstärken, vgl. z. B. L o e b ${ }^{16}$ ). Diese Ionen könnten gleichzeitig durch Influenzwirkung eine Änderung der Potentialverhältnisse der obersten Kathodenschicht hervorrufen, die eine Verminderung der Austrittsarbeit der Elektronen (z. B. beim Photoeffekt) zur Folge hat. Die Ionen können aber auch unmittelbar auf der Kathode selbst entstanden sein, z. B., nach einem Vorschlag von The in ${ }^{9}$, durch eine Dissoziation der Oxydmoleküle unter der Einwirkung der Gasentladung.

Welcher der 'genannten Vorgänge vorherrschend ist, kann noch nicht entschieden werden. Auch die Frage nach den physikalischen Vorgängen des Abklingens des Effektes und der Neutralisierung der Oberfläche ist noch recht ungeklärt. Wie man aus den Versuchen von The in ${ }^{9}$ schließen kann, handelt es sich dabei nur um Vorgänge auf der Kathode selbst; das Füllgas des Zählers hat auf die Neutralisierung keinen Einfluß. Tan a ka ${ }^{17}$ macht für die Neutralisierung bimolekulare Vorgänge verantwortlich und leitet unter Annahme einer dünnen isolierenden Oxydschicht die Emission eines allmählich abklingenden Elektronenstromes ab, gemäß $I=I_{0} / 1+\alpha t$ ( $\alpha=$ Konst.). In den Abb. 2 und 3 sind Abklingkurven nach dieser Formel eingezeichnet, die das zeitliche Abklingen des Effektes recht gut wiedergeben.

Die beschriebenen Versuche wurden im Sommer 1943 im Physikalischen Institut der Universität Köln durchgeführt. Hrn. Prof. K i r c h n e r möchte ich für sein Interesse an dem Problem auch an dieser Stelle vielmals danken.

${ }_{14}$ W. B. Nottingham, Physic. Rev. 39, 183 [1932] u. 49, 78 [1936].

15 P. L. Copeland, Physic. Rev. 57, 625 [1940].

${ }_{16}$ C. B. L o e b, Fund. Proc. of electr. discharge in Gases, Teil C, Abschn.6, Kap.X, S. 485-513, New York 1939.

${ }_{17} \mathrm{~T}$ a n aka, Proc. physic.-math. Soc. Japan 22, 899 [1940]. 